\title{
84. On Closed Subspaces of the Complete Ranked Spaces
}

\author{
By Hatsuo OKano
}

(Comm. by K. KunUGI, M.J.A., June 12, 1957)

The purpose of this note is to study the completeness of the subspaces of the complete ranked spaces. ${ }^{1)}$

Let $R$ be a ranked space. ${ }^{2} \quad$ For a subset $A, \bar{A}$ denotes the closure of $A$. And for a fundamental sequence $v=\left\{v_{\alpha}\left(p_{\alpha}\right), 0 \leq \alpha<\omega_{\mu}\right\}, \vartheta(v)$ denotes $\bigcap_{\alpha} I\left\{v_{\alpha}\left(p_{\alpha}\right)\right\}$ if $\omega_{\mu}<\omega_{\nu}$ and $\bigcap_{\alpha} v_{\alpha}\left(p_{\alpha}\right)$ if $\omega_{\mu}=\omega_{\nu}$. Now we shall introduce another topology as follows: for a subset $A$ of $R$, let $\widetilde{A}$ denote the set of all points $p$ such that $p \in \vartheta(v)$ for some fundamental sequences $v=\left\{v_{\alpha}\left(p_{\alpha}\right)\right\}$, where $p_{\alpha} \in A$. Then the following conditions are satisfied: ${ }^{3)}$

(1) $A \subseteq \tilde{A}$.

(2) If $A \subseteq B$, then $\widetilde{A} \subseteq \widetilde{B}$.

(3) $\widetilde{0}=0 .{ }^{4}$

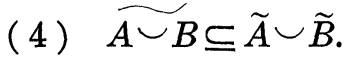

Take $\tilde{A}$ for the closure of $A$, and we get a new topology. We shall call it $r$-topology of $R$.

Let $S$ be a subset of $R$, then, for the usual relative topology, we have $\omega(S) \geq \omega(R)$. So $\omega_{\nu} \leq \omega(S)$. Hence we can take, as $\mathfrak{V}_{\alpha}\left(0 \leq \alpha<\omega_{\nu}\right)$, the set of all neighbourhoods of the form $v(p)=S \frown V(p)$, where $p \in S$ and $V(p) \in \mathfrak{B}_{\alpha}$ in $R$. Then the axiom (a) is satisfied and hence $S$ is a ranked space.

The closed (or $r$-closed) ${ }^{5)}$ subspaces of the complete ranked spaces are not always complete.

Example 1. Let $R$ be the set of all pairs $p=(x, y)$ of real numbers $x, y$. And let $E\left(n ; f_{1}, \cdots, f_{m}\right)$, where $m$ and $n$ are positive integers and $f_{i}(1 \leq i \leq m)$ is a straight line which passes over the origin $O=(0,0)$, denote the set of all points $p=(x, y)$ such that $x^{2}+y^{2}<\frac{1}{n^{2}}$ and $p \notin f_{i}$ for any $i$. Put $V\left(n ; f_{1}, \cdots, f_{m}\right)=\{O\} \smile E\left(n ; f_{1}, \cdots, f_{m}\right)$. The system of

1) See, for the notions and the terminologies, K. Kunugi: Sur les espaces complets et régulièrement complets. I, Proc. Japan Acad., 30, 553-556 (1954); and H. Okano: Some operations on the ranked spaces. I, Proc. Japan Acad., 33, 172-176 (1957).

2) Let the rank of $R$ be defined by $\omega_{\nu}$.

3) $\widetilde{\widetilde{A}} \subseteq \widetilde{A}$ is, in general, false. See, for example, H. Okano: Op. cit., Example 1. Cf. C. Kuratowski: Topologie, I, 20 (1948).

4) 0 denotes the empty set.

5) A subset is called $r$-closed if it is closed for $r$-topology. 
neighbourhoods of the origin is the family of all such $V\left(n ; f_{1}, \cdots, f_{m}\right)$ and the neighbourhoods of another point are given by the translation. Then we have $\omega(R)=\omega_{0}$. So we can put $\mathfrak{B}_{n}=$ the family of all neighbourhoods $V\left(n+1, f_{1}, \cdots, f_{m}\right)$ of all points. Then $R$ is a complete ranked space. Now by $S$ we shall denote the subspace of all points $p=(x, y)$ such that $y=0$ and $x \neq 0$. Then $S$ is closed for the given topology but not complete.

Example 2. Let $R$ be the same set as Example 1. And we shall give a topology as follows: for any positive integer $n, V_{n}$ denotes the set of all points $p=(x, y)$ such that $x^{2}+y^{2}<\frac{1}{n^{2}}$ or such that $x>0$ and $y=0$. The neighbourhoods of the origin are $\left\{V_{n}\right\}$ and, for another point, neighbourhoods are given by the translation. Then $\omega(R)=\omega_{0}$. So we put $\mathfrak{B}_{n}=$ the family of all neighbourhoods of type $V_{n+1}$ of all points. Then $R$ is complete. Denote by $S$ the subspace of all points such that $y=0$. Then $S$ is closed for the both topologies, that is $\bar{S}=\widetilde{S}=S$, but $S$ is not complete.

Now we shall give a sufficient condition for subspaces to be complete.

Lemma. Let $R$ be complete and $S$ be a subspace satisfying the conditions:

(1) If $p_{\alpha}\left(0 \leq \alpha<\gamma<\omega_{\nu}\right), q \in S, \bigcap_{\alpha} U_{\alpha}\left(p_{\alpha}\right) \cap S \supseteq V(q) \cap S$, sup $\gamma_{\alpha}<\gamma^{\prime}$ and $U_{\alpha}\left(p_{\alpha}\right) \in \mathfrak{B}_{\gamma_{\alpha}}, V(q) \in \mathfrak{B}_{\boldsymbol{r}^{\prime}}$ in $R$, then there exists $W(q)$ such that $W(q)$ $\in \mathfrak{B}_{\gamma^{\prime \prime}}$ in $R$, $\sup _{\alpha} \gamma_{\alpha}<\gamma^{\prime \prime} \leq \gamma^{\prime}, \bigcap_{\alpha} U_{\alpha}\left(p_{\alpha}\right) \supseteq W(q)$ and $W(q) \cap S \supseteq V(q) \cap S$.

(2) For any fundamental sequence $V=\left\{V_{\alpha}\left(p_{\alpha}\right)\right\}$ of $R$ such that $p_{\alpha} \in S$, we have $\vartheta(V) \cap S \neq 0$.

Then $S$ is complete.

Corollary. If $R$ is complete and $S$ is an $r$-closed subspace satisfying the condition (1) of the lemma, then $S$ is complete. 